\title{
NUMBER OF ZEROS OF A CERTAIN CLASS OF POLYNOMIALS IN A SPECIFIC REGION
}

\author{
N. A. Rather, Aijaz Bhat* and Liyaqat Ali
}

\begin{abstract}
We obtain results giving bounds concerning the number of zeros of polynomials with restricted coefficients in a specific region. Our results generalize and improve several well-known results concerning the number of zeros of polynomials in certain regions.
\end{abstract}

Mathematics subject classification (2020): 30C15, 30C10, 30E10.

Keywords and phrases: Polynomials, zeros, complex domain.

\section{REFERENCES}

[1] K. K. DEWAN, Extremal properties and coefficient estimates for polynomials with restricted zeros and on the location of zeros of polynomials, Ph. D. Thesis, Indian Institute of Technology, Delhi, 1980.

[2] N. K. Govil AND Q. I. Rahman, On the Eneström-Kakeya theorem II, Tohoku Math. J. 20 (1968) 126-136.

[3] Q. G. Mohammad, On the zeros of the polynomials, Amer. Math. Monthly 72 (1965) 631-633.

[4] NisAR A. RATHER, On some generalizations of Eneström-Kakeya theorem, J. Class. Anal. 14 (2019) 97-104.

[5] M. S. PUKHTA, On the zeros of a polynomial, Appl. Math. 2 (2011) 1356-1358.

[6] Q. I. Rahman, G. Schmeisser, Analytic Theory of Polynomials, Oxford University Press, (2002) 251-259.

[7] N. A. RATHER, IshfaQ DAR AND A. IQBAL, Generalization of Eneström-Kakeya theorem and its extension to analytic functions, J. Class. Anal. 16 (2020) 37-44.

[8] E. C. Titchmarsh, The Theory of Functions, 2nd Edition, Oxford University Press, London, (1939) 171. 